\title{
Calidad educativa universitaria en la zona de Planificación Ocho de Ecuador: Resignificación desde la investigación
}

\author{
University Educational Quality in the Eight Planning Zone of Ecuador: Resignification from Research
}

Qualidade do ensino universitário na área da Planificación Ocho do Equador: Resignificação da pesquisa

\section{ARTÍCULO DE INVESTIGACIÓN}

\section{Grace Elizabeth Escobar Medina gescobarm@ulvr.edu.ec \\ https://orcid.org/0000-0003-1171-5119}

\author{
María Leonor Cedeño Sempértegui \\ mcedenos@ulvr.edu.ec \\ https://orcid.org/0000-0002-7050-0671
}

\author{
Zoila Filomena Pesantez Cedeño \\ zpesantezc@ulvr.edu.ec \\ https://orcid.org/0000-0001-6701-5483
}

\author{
María Fernanda Chiriboga Posligua \\ mchiribogap@ulvr.edu.ec \\ https://orcid.org/0000-0002-0822-4485
}

Unidad de Posgrado, Facultad de Educación, Universidad Nacional Mayor de San Marcos, Ecuador

Recibido 24 de abril 2021 | Arbitrado y aceptado 9 de mayo 2021 | Publicado en 01 julio 2021

\section{RESUMEN}

Este artículo analiza las acciones humanas en la sociedad como producto integral y en particular a la educación considerada fuente de todo conocimiento y aprendizaje. Un rol social, basado en la calidad de la educación universitaria y el reposicionamiento de la función investigadora siendo el eslabón principal para emprender esta tarea. El objetivo de la investigación es provocar una resignificación de la investigación en el distrito 8 de Ecuador, contribuyendo a mejorar la calidad de la educación. El método es cualitativo y hermenéutico, se usó técnicas prácticas de entrevista a profesores y directivos del área de estudio. Los resultados indican que el hombre en constante cambio, se forma como profesional para una mejor calidad de vida y la investigación es el eje principal. Se concluye que los informantes coinciden en que el conocimiento científico está en construcción inicial y que la investigación debe estar interrelacionada con los resultados de aprendizajes.

Palabras clave: Investigación Educativa; Calidad Educativa Universitaria; Paradigma

\section{ABSTRACT}

This article analyzes human actions in society as an integral product and in particular education as the source of all knowledge and learning. A social role, based on the quality of university education and the repositioning of the research function as the main link to undertake this task. The objective of the research is to provoke a resignification of research in district 8 of Ecuador, contributing to improve the quality of education. The method is qualitative and hermeneutic, using practical interview techniques with teachers and directors of the area of study. The results indicate that the man in constant change, is formed as a professional for a better quality of life and research is the main axis. It is concluded that the informants agree that scientific knowledge is under initial construction and that research should be interrelated with learning outcomes.

Key words: Educational Research; University Educational Quality; Paradigm

\section{RESUMO}

Este artigo analisa as ações humanas na sociedade como um produto integral e em particular a educação como a fonte de todo o conhecimento e aprendizagem. Um papel social, baseado na qualidade da educação universitária e no reposicionamento da função de pesquisa como o principal elo para empreender esta tarefa. O objetivo da pesquisa é provocar uma re-significação da pesquisa no Distrito 8 do Equador, contribuindo para melhorar a qualidade da educação. O método é qualitativo e hermenêutico, utilizando técnicas práticas de entrevista com professores e gerentes na área de estudo. Os resultados indicam que o homem está em constante mudança, ele é treinado como profissional para uma melhor qualidade de vida e a pesquisa é o eixo principal. Concluise que os informantes concordam que o conhecimento científico está em construção inicial e que a pesquisa deve estar inter-relacionada com os resultados do aprendizado.

Palavras-chave: Pesquisa Educacional; Qualidade Educacional Universitária; Paradigma 


\section{INTRODUCCIÓN}

Este artículo de investigación es considerado de vital importancia, ya que aborda un tema de gran vigencia actual en la educación superior como lo es la calidad educativa. Al respecto, en la Agenda 2030 la cual tiene como objetivo el Desarrollo Sostenible emitida por la Comisión Económica para América Latina y el Caribe de las Naciones Unidad (2015) en donde refiere como esencia la calidad de los procesos educativos. Una educación de nivel adecuado que ayude a promover el conocimiento, creatividad y a su vez que garantice la consecución de las competencias básica, así como la resolución de problemas como claves, garantizando de esta forma que se lleven a cabo el desarrollo de los demás derechos fundamentales para la humanidad. (CEPAL, 2018).

Dentro de esta perspectiva, es oportuno señalar que desde el siglo XX, el estudio de la Calidad Educativa se ha visto incrementado. A tal efecto, a partir de la década de los 50 se da importancia significativa a la eficacia y eficiencia como indicadores que determinan el funcionamiento y el ejercicio de las entidades de enseñanza de nivel superior, así también el impacto tanto dentro y fuera de ellas, es decir, en los estudiantes, el entorno $y$ en el personal que en ellas labora.

La dinámica del desarrollo de la humanidad desde siempre, ha estado signada por el valor que ha adquirido el saber. A tal efecto, el conocimiento hoy, se caracteriza por los adelantos tecnológicos que cada día tienen un mayor impacto en la sociedad. Es por esto que se presenta la necesidad de que los establecimientos educativos, y especialmente las universidades, las cuales están llamadas a constituirse en el punto clave para generar nuevos y significativos conocimientos que determinen el éxito, y producción de las generaciones futuras. En este sentido, una de las aristas responsables de los cambios gestados en la humanidad lo constituye la investigación.
De manera tal que, Educación, Universidad e investigación, son consideradas las coordenadas para dar respuestas certeras a su entorno, y por ende, ofrecer una educación de calidad, entendida ésta, como un constructo pluridimensional en el que convergen múltiples factores, que a través de la indagación y el descubrimiento sistematizado, se solucionan problemas y se satisfacen necesidades.

En armonía con estas ideas, el acto investigativo en las universidades trascenderá los límites del desarrollo de un método científico riguroso, ya que la investigación vista desde esta concepción, se convierte en un simple requisito que aspira obtener datos que constituyen una parte de la realidad, para presentarlos en artículos científicos, que si bien es cierto son importantes en la consecución de las de las funciones de la Universidad, también lo es, el hecho de que no es un acto natural y cotidiano donde exista una comprensión holística de lo que acontece y el por qué sucede.

Se trata entonces de una resignificación del ejercicio propio de la investigación a nivel universitario, cuyo propósito sea el comprender la realidad y proponer alternativas y soluciones que sin duda conducen a su mejoramiento, y por ende optimizar la Calidad Educativa.

Sobre la base establecida en los párrafos anteriores, esta investigación persigue como objetivo analizar las acciones humanas y establecer el rol que tiene la función de la investigación en las instituciones de educación superior en la Zona 8 de Ecuador en el marco de la calidad educativa de dichos centros de educación.

\section{La investigación educativa}

$\mathrm{Si}$ se asume que actualmente el mundo gira en torno al redescubrimiento de la vida como una trama de relaciones auto-eco-organizadas, donde la complejidad Morín (2013) y la transdiscipinariedad (Nicolescau) emergen en contra de las posiciones reduccionistas colocadas en extremos: subjetividad, 
objetividad, deducción-inducción, explicacióncomprensión entre otras, la concepción de la investigación educativa puede abordarse como: la construcción de saberes en el ámbito educativo, a través de una vinculación epistémica entre el que quiere conocer y el que se muestra para ser conocido, donde uno no es uno sin la presencia del otro en un abanico de posibilidades que va desde la lógica objetiva-explicativa a la lógica subjetivacomprensiva que se despliega en un escenario transcomplejo educativo-social-cultural determinado.

En este devenir y configuración del investigar en el ámbito educativo, se nota con preocupación, como la investigación educativa se ha revestido de condiciones y elementos que lejos de acercarla a su esencia, las desvirtúan generando en la praxis toda suerte de falacias y mitos institucionalizados. Así, por ejemplo, investigar para muchos educadores, se reduce a realizar proyectos comunitarios, los cuales se identifican más con lo gerencial que con lo investigativo propiamente; de igual manera los proyectos centrados en el aula, por lo general, se limitan a copiar y reproducir esquemas y contenidos que en muchos casos no atienden a las necesidades de los actores sociales.

Si se considera que en materia de Investigación Educativa y fundamentalmente en lo que concierne a motivar, estimular y generar compromiso por parte del estudiante, el principal responsable es el docente, pero ¿ cómo dar lo que no se tiene? Esta frase pareciera un lugar común, pero es de importancia capital para el tema en desarrollo por cuanto en los procesos de formación docente los cuales se han venido observando que el currículo no responde al reto de formar un docente que a través de la investigación transforme su propia práctica. Con el respeto que merecen las universidades ecuatorianas, pareciera que investigar en los distintos programas de formación docente se reduce a presentar un informe de práctica profesional o un trabajo de grado, esto estimula "la creación de caldos de cultivo" para la reproducción de prácticas donde "enseñamos" a investigar cómo nos "enseñaron", a repetir viejos e improductivos esquemas, a trasladar a cualquier investigación, procedimientos divorciados de la naturaleza del fenómeno que se investiga y sin ninguna consideración epistémica reduciendo, por lo tanto, la investigación a una práctica meramente instrumental.

Las situaciones anteriormente descritas, conllevan a afirmar que la investigación educativa, dentro del marco universitario, en vez de ser un proceso orientado a generar saberes, aplicarlos, difundirlos, ampliarlos o innovar los ya existentes se ha convertido en lo que se ha denominado "Investigación plana", que no llega al estudiante, que no lo invita a asumir las cosas de manera distinta, que no articula la teoría con la praxis, que privilegia un solo modo de pensar y donde la transcomplejidad es el invitado de piedra.

Se puede observar que en las universidades ecuatorianas, no se atiende al proceso de investigación educativa, no existe la formación en los estudiantes de verdaderas competencias investigativas, la investigación sigue siendo una asignatura cuya queja fundamental, por parte de los estudiantes es que les "dan" materias aisladas y luego se exige un trabajo de grado, desarraigado, no contextualizado, lo cual trae angustia, tristeza y poco disfrute. Investigar es un mito, es una barrera.

Ante lo expuesto, se fija posición señalando que la investigación educativa en Ecuador no permite conocer la escuela en su más amplio sentido, por cuanto las producciones son desarraigadas, responden a intereses individuales $\mathrm{y}$ no organizacionales $\mathrm{y}$ en consecuencia poco pertinentes para explicar y comprender la dinámica sociocultural. Por otra parte, hay una pérdida de credibilidad en los trabajos deinvestigación producto de la autodesvalorización del propio educador hacia sus producciones a las cuales la única utilidad que le confieren es la de servir de vía para ascender en 
el escalafón o la obtención de un título académico, en detrimento del verdadero significado de su labor en la búsqueda de soluciones para transformar la realidad del proceso educacional.

Sin dudas, para todo ello se requiere de la debida inversión en el sector educativo superior, lo que a mediano y largo plazo tendrá un significado de relevancia por el aporte que constituyen las investigaciones universitarias para las empresas $y$, fundamentalmente, por el egresado más competente que se destinará a estas. Esta tendencia hacia la priorización de las investigaciones universitarias prevalece en los países de avanzada y debe tender a predominar en los países subdesarrollados, dada la presencia de la globalización.

En consecuencia, la aptitud científicoinvestigativa de la educación superior, se convierte en la mira principal de preocupaciones para responder a la formación de investigadores. Así, la aceptación del poder del conocimiento y las formas en que se produce, se apropia y evoluciona conduce a reorientar las funciones de la universidades, particularmente, en la región latinoamericana.

\section{La calidad en la investigación}

Las universidades deben considerar la distribución del horaria de los docentes investigadores, ya que si este es propuesto con actividades ligadas a otras dentro del quehacer universitario, poco o nada podrá aportar el docente investigador por producir, además, se hace necesario que las IES direccionen la ayuda económica con la importancia que ameritan todos los proyectos investigativos, que se considere también la investigación en lugares vulnerables con fondos de las IES. Es necesario considerar y garantizar la infraestructura de las universidades, según rango y especialidades de las investigaciones, se debe incluir la cobertura de los servicios investigativos en los centros de información y documentación.

\section{Educación e investigación universitaria en Ecuador}

La universidad en su condición de proceso encaminado a la producción de conocimientos útiles a la sociedad $\mathrm{y}$ al enriquecimiento de la ciencia, permite dar respuestas a vacíos del conocer y construye una vía que dado su sustento científico coadyuva al avance tecnológico y por ende al desarrollo social. Las universidades de Ecuador han enfrentado en las últimas décadas desde el año 2009, 2012, 2013 y 2015, algunos procesos evaluativos, los mismos que han estado a cargo de diferentes instancias de turno siendo la primera CEAACES (Consejo de evaluación acreditación y aseguramiento de la calidad de la educación superior), actualmente CACES (Consejo de aseguramiento de la calidad de la educación superior).

En los últimos años, en Ecuador se viene manifestando un notable avance y progreso en materia de investigación científica a nivel de las instituciones universitarias. Todo ello obedece, a que en la etapa de la revolución ciudadana, se puso mayor interés y se trabajó estratégicamente en elevar los estándares académicos. A partir del año 2008, se pone en marcha un modelo educativo para el sector universitario, centrado en la investigación científica, a fin de superar las insuficiencias existentes y poder repotenciar la educación ecuatoriana. Al respecto Ecuador desde el año 2018 entro en convenio con Alemania para coordinar acciones que busquen impulsar la academia con la industria.

La producción de conocimiento emerge de una multiplicidad de centros e instituciones, no obstante, las universidades son protagonistas en el desarrollo de las investigaciones, inclusive desde su concepción y el liderazgo de proyectos de gran impacto para la sociedad en general. En las universidades se encuentra el capital humano preparado para conducir los procesos de búsqueda 
y producción del conocimiento, orientando la labor que, al respecto, pudieran tener investigadores que inician su trayectoria y que desean contribuir de manera significativa al progreso de la nación. De esta manera, se tendrán posibilidades certeras y diversas para el enriquecimiento de los sistemas productivos, obtención de información novedosa que permita generar innovaciones en diferentes campos disciplinares y, por supuesto, la consecución de recursos financieros para proyectos de gran impacto y retorno potencial.

Sin embargo, ante los avances logrados, en la práctica, en el día a día de las universidades, los resultados no alcanzaron los discretos niveles esperados, debido, en parte, a la masificación de la educación en establecimientos públicos que trajo consigo, un gran contingente de estudiantes que ingresaron a las universidades y que demandaban una fuerte atención por parte de los docentes, quienes tuvieron que dedicar más tiempo a la preparación metodológica y la docencia ante grupos numerosos de estudiantes, con pocas posibilidades de tiempo para la investigación; además desmotivados para todas las actividades, producto de que la remuneración recibida no compensa los esfuerzos.

Las carencias y limitaciones del sistema para la educación superior constituyen obstáculos para asumir problemas estructurales de la sociedad y emprender la búsqueda de soluciones sustentadas científicamente por procesos investigativos. Hasta entonces, la innovación académica y la cultura investigativa sólo constituían una pretensión. Esta afirmación, es corroborada por un estudio efectuado por la Universidad Técnica de Ambato, en el periodo 1983 a 2008, dicho estudio analiza la producción científica de esta casa de estudios, de lo cual resultó que solo el 50 por ciento de las facultades de esa institución evidenció niveles participativos en proyectos investigativos y de producción científica.

En base a los considerado, en Ecuador, la ruta iniciada en el contexto de la investigación cuenta con múltiples inconvenientes que deben ser vencidos, desde la dinámica interna las IES, deben orientarse hacia recuperar la credibilidad mantenida, la calidad y solvencia científica, empezando por que la misión y visión institucional según el marco social sea orientada de acuerdo a sus menesteres y exigencias los mismos que versan en físicamente en el documento cuyo nombre es "Desarrollo del Vínculo de Universidad- Sociedad en el Ecuador".

Como se puede apreciar, la educación superior ecuatoriana requiere una resignificación de su quehacer, orientada a la inclusión y vinculo del componente investigativo en el proceso en la formación profesional con la finalidad de hacer más pertinente la gestión educativa, adecuándola a las demandas de los nuevos tiempos y formando a un egresado con las competencias indispensables para poder hacer frente a las exigencias de su entorno social, en donde los cambios en materia económica, educativa, social, política y cultural, requieren de un ciudadano plenamente preparado para dar respuesta a los desafíos y los avances en los contextos científicos, tecnológicos y comunicacionales.

\section{MÉTODOS}

Metodológicamente la investigación se sustentó desde las perspectivas (Epistémica, ontológica y axiológica). Por tanto, es cualitativo y hermenéutico. Para realizar el proceso de la investigación se determinaron cinco universidades de la zona 8 se entrevistó a 60 docentes que cumplían la función de coordinadores de carreras para conocer la situación actual del proceso de evaluación, así como también visualizar a nivel de universidades el escenario del proceso investigativo. Para ello se utilizaron técnicas prácticas de entrevista a profesores y directivos del área de estudio, los resultados se analizan mediante técnicas de clasificación, estructuración y comparación. Se pudo determinar, que en su gran mayoría las universidades tenían el mismo problema para cumplir con el indicador de investigación, el 
común de este es relativamente bajo ya que no se cuenta con información requerida por el CES sobre el criterio investigación, que consiste en determinado número de artículos, libros y publicaciones que debe justificar la carrera, además de tener definidas las líneas de investigación formativa y cuando sea posible y pertinente de investigación básica, la exigencia requería que los trabajos de titulación se enmarcaran en dichas líneas.

Así mismo, dicha selección de universidades de la zona 8 , se base en el hecho de que se encuentran en proceso de evaluación y acreditación, esto ayudó a obtener la información a través de la coordinación de cada una de las carreras las mismas que debían cumplir con los indicadores de calidad dentro de un tiempo determinado, para esto se requería subir a la plataforma la información determinada por el criterio y el indicador. Respecto al criterio investigación la pertinencia corresponde a las publicaciones de libros y revista revisadas y no revisadas así como sus resultados, los datos obtenidos en las universidades observadas corresponden a criterios de resultados muy bajos.

\section{RESULTADOS Y DISCUSIÓN}

En este epígrafe se reflejan los hallazgos investigativos logrados a través de la exploración realizada en las Universidades de la Zona 8 de Ecuador. Fue importante recabar información emana de los docentes coordinadores de carreras mediante la técnica de entrevista. Se obtuvo la categorización, para lo que se tomó como referencia la definición de Strauss y Corbin (2002) que plantea: "la asignación de conceptos a un nivel más abstracto (...) las categorías tiene un poder conceptual puesto que tienen la capacidad de reunir grupos de conceptos o subcategorías" (p.2). Así, Martínez (2012) refiere: "la categoría resume lo que el informante dice ya que es la concreta de la información obtenida" (p.101). Los resultados acerca de la categorización por cada informante, se ofrecen, dada su extensión en la siguiente Tabla 1: 
Tabla 1. Hallazgos presentados a través de las tres categorías desarrolladas sobre la calidad educativa.

\section{CATEGORÍA}

Debilidades

Investigativas

"rasgos creo que han venido subiendo mucho de categorías podemos decir o de niveles más bien, iniciamos de pronto con un nivel un poco empírico en la universidad en el que de pronto el mismo hecho de que querer aprender más nos ponía en un sitio de curiosidad pero a medida que fue pasando el tiempo el tipo de investigación ya fue mejor encaminada de pronto el mismo hecho que no teníamos eso parámetros o la metodología verdad que verdaderamente necesita un investigador verdad era un nivel más bajo ósea en nivel 1 actualmente podríamos decir nosotros que el nivel es mucho más alto porque la experiencia que se ha venido acumulando"

Mejoras

Continuas "el 2012 lo veo que es un año trascendental porque justamente es el año en qué en esta universidad ...existe un departamento de investigación que es cuando se empieza la acogida de docentes de investigadores a tiempo completo ... somos investigadores siempre hemos sido ... fuimos de pronto los primeros docentes que tuvimos artículos ... los pioneros ... ahora las cuestión de las ponencias ... una visión dentro de la ciudad dentro de un país ahora ... miras al exterior para hacer ponencias internacionales ... artículo escribirlo ...valor intelectual ... tener un libro ... aspiraciones que tenemos van más allá como investigadores y esto ha servido para también llevarlo al aula y trasmitirlo al estudiante sobre todo cuando uno dirige tesis .... estar al día en los cuestionamientos de normas APA ... investigador porque el investigador más allá de siempre estar habido de querer aprender más estar siempre dispuesto a trascender dejando un legado"

TÉCNICAS

DOCUMENTAL

OBSERVACIÓN

Rosas y otros (2006), sostienen que se han realizado varios estudios a nivel nacional e internacional, que demuestran la baja productividad en materia de investigación universitaria; por una parte, es debido a una supervisión extemporánea, además de otras variables que convergen igualmente.

Aunque esta es una situación humana, multifactorial, se ha detectado que la carencia de competencias investigativas influyen de forma negativa en las experiencias investigativas.

Hermoso (2010), sostiene que el hombre está en constante cambio y se dirige hacia la búsqueda de información relevante para su existencia la cual influye en la transformación de sus saberes. En este sentido, se instruye y se forma como profesional en un área de interés, donde puede ser partícipe de situaciones, que le generen bienestar y una mejor calidad de vida
"Según información suministrada, existen debilidades marcadas en la función de investigación en la universidad. Se observa sin embargo de acuerdo a los hallazgos de la entrevista y lo observado por la investigadora, el deseo de superarlas y establecer como prioritario este campo que en opinión de la entrevistada recién se inicia en la institución".

El compromiso hacia la calidad y la investigación es un hecho que plasma a través de sus palabras la docente entrevistada. De acuerdo con lo expresado esta casa de estudio de nivel superior siempre ha estado a la vanguardia de dar respuesta a la colectividad a través de la investigación. De allí que en su opinión, desde hace algunos años se ha venido considerando la importancia de mejoras continuas, y la investigación ha constituido la herramienta para lograrlo" 


\begin{tabular}{|c|c|c|c|}
\hline \multirow{2}{*}{ CATEGORÍA } & \multicolumn{3}{|c|}{ TÉCNICAS } \\
\hline & ENTREVISTA EN PROFUNDIDAD & DOCUMENTAL & OBSERVACIÓN \\
\hline $\begin{array}{l}\text { Desarrollo de } \\
\text { Competencias } \\
\text { Investigativas }\end{array}$ & $\begin{array}{l}\text { "Eleva la Calidad Académica, pues contar con Docentes } \\
\text { Investigadores no solo serviría para obtener un buen puntaje en } \\
\text { los indicadores de evaluación con fines de acreditación y obtener } \\
\text { una categorización privilegiada para nuestra Universidad, sino que } \\
\text { transmitimos esas habilidades científicas a nuestros estudiantes y } \\
\text { en consecuencia tendremos un producto de primer nivel, hablando } \\
\text { de estudiantes como profesionales también investigadores que } \\
\text { aportarán con sus conocimientos al país y al mundo" }\end{array}$ & $\begin{array}{l}\text { Sánchez ( } 2008 \text { ), dice que el investigador crítico } \\
\text { es aquel que se inscribe en una modalidad } \\
\text { investigativa centrada en la construcción crítica del } \\
\text { conocimiento como posibilidad para la generación } \\
\text { de proyectos emancipatorios, enmarcados en } \\
\text { una praxis transformadora, que rompe con la } \\
\text { racionalidad dominante, instrumental, utilitarista, } \\
\text { individualista y productivista. Así, reconocer } \\
\text { y repensar la realidad requiere que la misma } \\
\text { sea percibida en forma holística, captando la } \\
\text { complejidad humana y sus manifestaciones en } \\
\text { todos los acontecimientos relacionados con la } \\
\text { sociedad. }\end{array}$ & $\begin{array}{l}\text { "Se observa la convicción del papel } \\
\text { protagónico que tiene la investigación en } \\
\text { el logro de la calidad educativa. se destaca } \\
\text { el hecho de que a través de la investigación } \\
\text { se puede desarrollar habilidades científicas } \\
\text { tanto de docentes como de los estudiantes" }\end{array}$ \\
\hline
\end{tabular}




\section{Discusión}

Esta investigación tuvo su esencia en el ser, y en el profesional que egresa de las IES en Ecuador, y de manera particular las pertenecientes a la Zona 8. Mejorar la calidad educativa fue el horizonte, y el enfoque cualitativo el camino para el éxito del estudio. Lo más importante es la interacción desde lo humano a través de entrevistas con los informantes, lo que permitió conocer desde lo natural mediante las bondades del método hermenéutico, la realidad acerca de la concepción que éstos profesionales tienen sobre la investigación y la calidad educativa.

Para Pérez (2015), quien en su trabajo sobre las "Percepciones sobre la tesis de grado en profesores y alumnos de la universidad", muestra resultados del desarrollo del trabajo de grado en el contexto seleccionado y contando con los criterios de estudiantes y docentes. En síntesis aborda las motivaciones que le indujeron al proceso investigativo con la debida consulta bibliográfica acerca de la temática de literatura perteneciente a España, de donde procede el estudio, así como las experiencias de otros países, todo lo cual le posibilitó asumir una actitud ante la formulación del tema y de ahí la formulación del problema a atender. Muestra resultados considerando los criterios de estudiantes y docentes; aborda las motivaciones en el proceso investigativo. El mismo que coincidimos en la imperiosa necesidad de promover estrategias que motiven la investigación y que no sea motivo de limitaciones de estudio.

Espinoza (2014), en su tesis, "El Perfil del docente Investigador", señala su experiencia en la formación de investigadores al nivel de los IES, expone los componentes relacionados al perfil del investigador de acuerdo al contexto que estudia y define los antecedentes de mayor utilidad. Del mismo se consideró la necesidad de fomentar la labor investigativa asociada al tema y las disciplinas a través de las cuales se puede atender el quehacer del docente en ese orden; además propone las cualidades que debe exhibir el investigador, desde una mirada humana, científico y técnico.

Los estudios consultados representan experiencias desde lo teórico y lo práctico y buscan dar solución a las debilidades que se presenten la investigación en el contexto universitario. Los autores referidos coinciden en señalar que la investigación constituye una función que debe re-significada a fin de lograr radicar actitudes improductivas en los estudiantes con especial atención en los niveles de postgrado y deja ver la necesidad imperiosa de efectuar una gestión orientada a optimizar la calidad educativa.

\section{CONCLUSIONES}

En base a las ideas iniciales para el mejoramiento de la calidad educativa universitaria a partir de la resignificación de la investigación. Se concluye:

- La calidad educativa a la luz de este estudio se concibe como nueva perspectiva del quehacer universitario, por ello requiere del rol protagónico de las IES como actores claves en el desarrollo y consolidación de la investigación universitaria.

- La calidad es un parámetro importante en educación, por ello es necesario resignificar la investigación considerando parámetros actuales e internacionales.

- La investigación en las IES se encuentra en construcción, por ello se considera tomar en cuenta los cambios a favor de la investigación que ha realizado el Ecuador en estos últimos diez años.

\section{REFERENCIAS}

CACES (2019). Modelo de evaluación externa de universidades y escuelas politécnicas 2019. Consejo de Aseguramiento de la Calidad de la Educación Superior 
CEAACES, modelo genérico de evaluación del entorno de aprendizaje de carreras en Ecuador, (comisión permanente de evaluación de carreras) versión: 2011, 2013, 2015, 2016, 2017, 2018

CEPAL (2018) La Digitalización industrial, un camino hacia la gobernanza colaborativa

Espinoza, A. (2014). El Perfil del docente Investigador. (Tesis Doctoral). Universidad Autónoma de Tlaxcala/ Universidad Autónoma del estado de Hidalgo

Hermoso, V. (2010). Filosofía: una matriz cultural. Maracay: Ediciones de la fundación Cultural y tradición

Martínez M. (2012). Ciencia y arte en la metodología cualitativa.: Trillas

Morín, (2013) E. (s.f.). La complejidad y la acción. Universidad Tecnológica de Pereira. http:// www.utp.edu.co/rectoria/la-complejidad-y-laaccion-edgar-morin.pdf
Pérez, A. (2015). Percepciones Sobre la Tesis de Grado En Profesores y Alumnos de La Universidad. Síntesis de una Investigación. (Tesis doctoral). Universidad de Vigo

Rosas y Otros. (2006). Rol del Tutor de Tesis: Competencias, condiciones personales y funciones. Investigación y Postgrado. http:// www.scielo.org.ve/scielo.php?Pid=S1316$00872006000100007 \&$ script $=$ sci_arttext

Sánchez, S. (2008).Fundamentos para la Investigación Educativa. Presupuestos epistemológicos que orientan la investigación. Cooperativa. Editorial Magisterio

Strauss, A., and Corbin, J. (2002). Basics of qualitative research: Techniques and procedures for developing grounded theory (2nd Ed.). Thousand Oaks, CA: Sage 